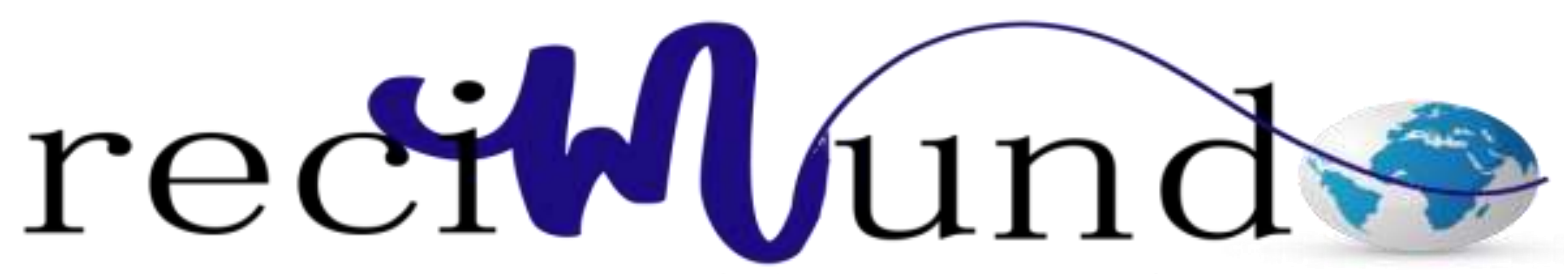

Revista Cientifica Mundo de la Investigación y el Conocimiento

Erick Hugo Zambrano Franco ${ }^{\text {a; }}$ Rossibell Berenisse Ollague Armijos ${ }^{\text {b; }}$ Betsy Elizabeth Quisilay Guamán ${ }^{\text {c; }}$ Lenin Israel Velasco Chávez ${ }^{\text {d }}$

Mecanismos de las arritmias cardíacas

Mechanisms of cardiac arrhythmia

Revista Científica Mundo de la Investigación y el Conocimiento. Vol. 3 núm.3, septiembre, ISSN: 2588-073X, 2019, pp. 717-734

DOI: $10.26820 /$ recimundo/3.(3).septiembre.2019.717-734

URL: http://recimundo.com/index.php/es/article/view/546

Código UNESCO: 3205 Medicina Interna

Tipo de Investigación: Artículo de Revisión

Editorial Saberes del Conocimiento

Recibido: 15/05/2019

Aceptado: 23/06/2019

Publicado: 30/09/2019

Correspondencia: $\underline{\text { dr_ezambrano@hotmail.com }}$

a. Médico; Investigador Independiente; Guayaquil, Ecuador; dr_ezambrano@ hotmail.com

b. Médico; Investigador Independiente; Guayaquil, Ecuador; dra ollague@ hotmail.com

c. Médico; Investigador Independiente; Guayaquil, Ecuador; betsyquisilayguaman@gmail.com

d. Médico; Investigador Independiente; Guayaquil, Ecuador; isra_barsa17@yahoo.es 


\section{Mecanismos de las arritmias cardíacas}

Vol. 3, núm. 3., (2019)

Erick Hugo Zambrano Franco; Rossibell Berenisse Ollague Armijos; Betsy Elizabeth Quisilay Guamán; Lenin Israel Velasco Chávez

\section{RESUMEN}

La circulación sanguínea es el resultado de los latidos del corazón, que proporciona la fuerza mecánica para bombear la sangre oxigenada hacía, y la sangre desoxigenada lejos de, los tejidos periféricos. Esto depende críticamente de la activación eléctrica anterior. Las interrupciones en el patrón ordenado de esta onda de excitación cardiaca pueden conducir a arritmias. La comprensión de los mecanismos que subyacen a su generación y mantenimiento requiere el conocimiento de las contribuciones iónicos para el potencial de acción cardíaco, que se discute en esta revisión. Un breve resumen de las diferentes clasificaciones, se proporcionan sistemas de cationes para arritmogénesis, seguido de una discusión detallada de cada mecanismo a su vez, destacando los avances recientes en esta área.

Palabras Claves: Arritmia; Circulación sanguínea; Acción cardiaca. 


\title{
Mecanismos de las arritmias cardíacas
}

Vol. 3, núm. 3., (2019)

Erick Hugo Zambrano Franco; Rossibell Berenisse Ollague Armijos; Betsy Elizabeth Quisilay Guamán; Lenin Israel Velasco Chávez

\begin{abstract}
Blood circulation is the result of the heartbeat, which provides the mechanical force to pump the oxygenated blood to, and the deoxygenated blood away from, the peripheral tissues. This critically depends on the previous electrical activation. Interruptions in the orderly pattern of this wave of cardiac excitation can lead to arrhythmias. Understanding the mechanisms that underlie its generation and maintenance requires knowledge of ionic contributions to the potential for cardiac action, which is discussed in this review. A brief summary of the different classifications, cation systems for arrhythmogenesis are provided, followed by a detailed discussion of each mechanism in turn, highlighting recent advances in this area.
\end{abstract}

Key Words: Arrhythmia; Blood circulation; Cardiac action.

\section{Introducción.}




\section{Mecanismos de las arritmias cardíacas}

Vol. 3, núm. 3., (2019)

Erick Hugo Zambrano Franco; Rossibell Berenisse Ollague Armijos; Betsy Elizabeth Quisilay Guamán; Lenin Israel Velasco Chávez

El latido del corazón proporciona la fuerza mecánica para el bombeo de la sangre oxigenada a, y la sangre desoxigenada lejos de los tejidos periféricos. Esto depende críticamente de la activación ordenada y la recuperación de la excitación eléctrica a través del miocardio.

Las interrupciones de esto pueden conducir a arritmias. La comprensión de los mecanismos que subyacen a su generación y mantenimiento requiere el conocimiento de las contribuciones iónicos al potencial de acción celular, que se discute en esta revisión. Un breve resumen de los diferentes clasificaciónfia continuación, se proporcionan sistemas de cationes de arritmogénesis, seguidos por una discusión de cada mecanismo a su vez, destacando los avances recientes en esta área.

El potencial de acción cardíaco resultados de la secuencia abierta y el cierre de proteínas de los canales de iones que atraviesan la membrana plasmática de los miocitos individuales. Su conducción a través del corazón depende de acoplamiento eléctrico entre estas células, que está mediada por uniones comunicantes (Kanno \& Saffitz, 2001). Las diferencias en la expresión y propiedades de los canales de iones dan lugar a heterogeneidades en la acción posibles formas de onda en diferentes regiones cardiacas y tipos de células, y en la propagación unidireccional normal de los potenciales de acción a través del corazón. El potencial de acción cardíaco en el ser humano tiene cinco diferentes fases (de 0 a 4). La despolarización de la SA nodo trae el potencial de membrana con el umbral, la apertura de los canales de sodio activados por voltaje (Kunze, Lacerda, \& Wilson, 1985). Esto permite que los iones de sodio se difundan hacia abajo de su gradiente electroquímico del espacio extracelular, a través de la membrana y en la célula. La corriente de sodio resultante, IN / A, produce un bucle de retroalimentación positiva que hace que más canales de sodio se abran, y la despolarización de la membrana continúa hasta que se 


\section{Mecanismos de las arritmias cardíacas}

Vol. 3, núm. 3., (2019)

Erick Hugo Zambrano Franco; Rossibell Berenisse Ollague Armijos; Betsy Elizabeth Quisilay Guamán; Lenin Israel Velasco Chávez

alcanza el potencial de Nernst o cuando los canales son inactivados. Esta es responsable de la rápida carrera ascendente, denominado fase 0 , del potencial de acción.

La repolarización temprana y rápida resulta de la activación de las corrientes de salida de potasio transitorios rápidos y lentos, Itof y Itos, y es responsable de la fase 1 del potencial de acción. Esto es seguido por una meseta prolongada, debido a un equilibrio entre las corrientes de entrada mediadas por el canal de calcio tipo L dependientes de voltaje (ICalifornia) Y sodiointercambiador de calcio (INCX), Y las corrientes hacia el exterior mediadas por el rectificador retrasado por voltajeficanales de potasio (IK) (Carmeliet, 1999). Las corrientes rápidas y lentas (IKr y yoKansas, respectivamente) constituyen IK. También hay contribución de la rectificación de la corriente de entrada (IK1). Esta meseta es responsable de la fase 2 del potencial de acción. La fuerza impulsora para el eflujo de potasio se mantiene alta durante la fase de meseta debido a una gran diferencia entre el potencial de membrana y el potencial Nernst de potasio. Como los canales de calcio se convierten en inactivados, las corrientes de salida de potasio predominan, causando más repolarización y con el potencial de membrana hacia el potencial de equilibrio de potasio. Este es el responsable de la fase 3 del potencial de acción.

El potencial de membrana vuelve a su valor de reposo después de completada la repolarización, que corresponde a la fase 4 del potencial de acción, y normalmente se polariza a valores de entre 80 y $64 \mathrm{mV}$ con respecto al espacio extracelular (Amos, Wettwer, \& Metzger, 1996). Este estado de reposo se mantiene principalmente por los rectos hacia adentrofiactual, Ik1. La débil rectificación hacia el interior de los canales de potasio dependientes de ATP (Ik,atp), activado por difosfatos de nucleótido e inhibida por el trifosfato de adenosina, también son activos durante esta fase. Se cree que proporcionar un vínculo entre el metabolismo celular y 


\section{Mecanismos de las arritmias cardíacas}

Vol. 3, núm. 3., (2019)

Erick Hugo Zambrano Franco; Rossibell Berenisse Ollague Armijos; Betsy Elizabeth Quisilay Guamán; Lenin Israel Velasco Chávez

el potencial de membrana celular en marcapasos son distintos de otros tipos de células en mostrar automatismo, una propiedad resultante tanto por voltaje y mecanismos calcio dependientes. El primero consiste en la divertida corriente (If) llevado por canales de nucleótidos cíclicos activado por hiperpolarización $(\mathrm{HCN})$, que tienen varias características inusuales, tales como la activación en la hiperpolarización, permeabilidad a los iones de sodio y potasio, la modulación por AMP cíclico intracelular, y una pequeña conductancia de un solo canal. Esta última implica liberación espontánea de calcio desde el retículo sarcoplásmico, que activa Incx. Su papel fundamental se demostró en ratones con fibrilación completa específica knockout de NCX, que no mostró ninguna actividad de marcapaso. Ambos mecanismos dan lugar a la despolarización espontánea que es responsable de la pendiente ascendente del potencial de membrana.

\section{Metodología.}

Para el desarrollo de este proceso investigativo, se plantea como metodología la encaminada hacia una orientación científica particular que se encuentra determinada por la necesidad de indagar en forma precisa y coherente una situación, en tal sentido (Davila, 2015) define la metodología "como aquellos pasos previos que son seleccionados por el investigador para lograr resultados favorables que le ayuden a plantear nuevas ideas".(p.66)

Lo citado por el autor, lleva a entender que el desarrollo de la acción investigativa busca simplemente coordinar acciones enmarcadas en una revisión bibliográfica con el fin de complementar ideas previas relacionadas a los mecanismos de la arritmia cardiaca a través de 


\section{Mecanismos de las arritmias cardíacas}

Vol. 3, núm. 3., (2019)

Erick Hugo Zambrano Franco; Rossibell Berenisse Ollague Armijos; Betsy Elizabeth Quisilay Guamán; Lenin Israel Velasco Chávez

una revisión de literatura, para así finalmente elaborar un cuerpo de consideraciones generales que ayuden a ampliar el interés propuesto.

Tipo de Investigación

Dentro de toda práctica investigativa, se precisan acciones de carácter metodológico mediante las cuales, se logra conocer y proyectar los eventos posibles que la determinan, así como las características que hacen del acto científico un proceso interactivo ajustado a una realidad posible de ser interpretada. En este sentido, se puede decir, que la presente investigación corresponde al tipo documental, definido por Castro (2016), "se ocupa del estudio de problemas planteados a nivel teórico, la información requerida para abordarlos se encuentra básicamente en materiales impresos, audiovisuales y /o electrónicos”. (p.41).

En consideración a esta definición, la orientación metodológica permitió la oportunidad de cumplir con una serie de actividades inherentes a la revisión y lectura de diversos documentos donde se encontraron ideas explicitas relacionadas con los tópicos encargados de identificar a cada característica insertada en el estudio. Por lo tanto, se realizaron continuas interpretaciones con el claro propósito de revisar aquellas apreciaciones o investigaciones propuestas por diferentes investigadores relacionadas con el tema de interés, para luego dar la respectiva argumentación a los planteamientos, en función a las necesidades encontradas en la indagación.

\section{Fuentes Documentales}

El análisis correspondiente a las características que predomina en el tema seleccionado, llevan a incluir diferentes fuentes documentales encargadas de darle el respectivo apoyo y en ese 


\section{Mecanismos de las arritmias cardíacas}

Vol. 3, núm. 3., (2019)

Erick Hugo Zambrano Franco; Rossibell Berenisse Ollague Armijos; Betsy Elizabeth Quisilay Guamán; Lenin Israel Velasco Chávez

sentido cumplir con la valoración de los hechos a fin de generar nuevos criterios que sirven de referencia a otros procesos investigativos. Para (CASTRO, 2016) las fuentes documentales incorporadas en la investigación documental o bibliográfica, "representa la suma de materiales sistemáticos que son revisados en forma rigurosa y profunda para llegar a un análisis del fenómeno".(p.41). Por lo tanto, se procedió a cumplir con la realización de una lectura previa determinada para encontrar aquellos aspectos estrechamente vinculados con el tema, con el fin de explicar mediante un desarrollo las respectivas apreciaciones generales de importancia.

\section{Técnicas para la Recolección de la Información}

La conducción de la investigación para ser realizada en función a las particularidades que determinan a los estudios documentales, tiene como fin el desarrollo de un conjunto de acciones encargadas de llevar a la selección de técnicas estrechamente vinculadas con las características del estudio. En tal sentido, (Bolívar, 2015), refiere, que es "una técnica particular para aportar ayuda a los procedimientos de selección de las ideas primarias y secundarias”. (p. 71).

Por ello, se procedió a la utilización del subrayado, resúmenes, fichaje, como parte básica para la revisión y selección de los documentos que presentan el contenido teórico. Es decir, que mediante la aplicación de estas técnicas se pudo llegar a recoger informaciones en cuanto a la revisión bibliográfica de los diversos elementos encargados de orientar el proceso de investigación. Tal como lo expresa, (Bolívar, 2015) "las técnicas documentales proporcionan las herramientas esenciales y determinantes para responder a los objetivos formulados y llegar a resultados efectivos" (p. 58). Es decir, para responder con eficiencia a las necesidades investigativas, se introdujeron como técnica de recolección el método inductivo, que hizo posible 


\section{Mecanismos de las arritmias cardíacas}

Vol. 3, núm. 3., (2019)

Erick Hugo Zambrano Franco; Rossibell Berenisse Ollague Armijos; Betsy Elizabeth Quisilay Guamán; Lenin Israel Velasco Chávez

llevar a cabo una valoración de los hechos de forma particular para llegar a la explicación desde una visión general.

Asimismo, se emplearon las técnicas de análisis de información para la realización de la investigación que fue ejecutada bajo la dinámica de aplicar diversos elementos encargados de determinar el camino a recorrer por el estudio, según, (Bolívar, 2015) las técnicas de procesamiento de datos en los estudios documentales "son las encargadas de ofrecer al investigador la visión o pasos que debe cumplir durante su ejercicio, cada una de ellas debe estar en correspondencia con el nivel a emplear" (p. 123). Esto indica, que para llevar a cabo el procesamiento de los datos obtenidos una vez aplicado las técnicas seleccionadas, tales como: fichas de resumen, textual, registros descriptivos entre otros, los mismos se deben ajustar al nivel que ha sido seleccionado.

\section{Resultados.}

Mecanismos de arritmias

Varios esquemas se han utilizado para clasificar los mecanismos de las arritmias cardiacas. Tradicionalmente, estos han sido divididos en actividades de no entrante y reentrada. Un esquema alternativo los dividió en los que se producen en los niveles celulares y tisulares. Una clasificación basada en la dinámica, centrándose en las interacciones sustrato gatillo de tejidos, mecanismos arritmogénicos divididos en el ciclo inestable de calcio, reducción de la reserva de repolarización, y el exceso de reserva de repolarización (Weiss, Garfinkel, \& Karagueuzian, 2015). 


\section{Mecanismos de las arritmias cardíacas}

Vol. 3, núm. 3., (2019)

Erick Hugo Zambrano Franco; Rossibell Berenisse Ollague Armijos; Betsy Elizabeth Quisilay Guamán; Lenin Israel Velasco Chávez

\section{La actividad focal}

La actividad Focal puede surgir de automaticidad mejorada o actividad disparada o accionada (Figura 1).

\section{Automaticidad mejorada}

Las células marcapasos están presentes en el nodo $\mathrm{SA}$, aurículas, el nodo AV y el sistema HisPurkinje. En el corazón humano, la tasa normal de descarga del nodo SA es de entre 60 y 100 latidos por min (bpm). Marcapasos subsidiarios descargan a tasas más lentas. Por lo general son latente y restablecen por el marcapasos dominante con la tasa intrínseca más alta de la descarga (es decir, el nodo SA). Por ejemplo, el nodo AV se descarga en 40-60 bpm y el sistema de Purkinje descargas a 20-40 lpm. un incremento del automatismo de las células marcapasos puede aumentar la velocidad de descarga de la acción potencial (Figura 1). Esto puede resultar de tres mecanismos principales: un cambio negativo en el umbral del potencial (TP, flecha superior roto), un cambio positivo en el potencial diastólico máximo (MDP, flecha quebrada parte inferior), y un aumento de la frecuencia de la fase 4 de despolarización (Jalife, Delmar, \& Anumonwo, 2009). Cuando esto ocurre en el nodo SA, que puede conducir a un aumento de la frecuencia cardíaca, denominado taquicardia sinusal. Esto puede ser fisiológica, debido al aumento del tono simpatético durante el ejercicio, o fisiopatológico, debido a la hipovolemia, isquemia, o trastornos electrolíticos. Por otra parte, el síndrome de taquicardia-bradicardia ha sido visto en pacientes con fibrilación alterna fisíndrome de los ganglios fibrilación y del seno enfermo. Sus mecanismos moleculares subyacentes no han sido completamente aclarada. La evidencia reciente sugiere un posible papel de la regulación a la baja de $\mathrm{HCN}$ canal en el nódulo 


\section{Mecanismos de las arritmias cardíacas}

Vol. 3, núm. 3., (2019)

Erick Hugo Zambrano Franco; Rossibell Berenisse Ollague Armijos; Betsy Elizabeth Quisilay Guamán; Lenin Israel Velasco Chávez

SA, con la consiguiente disminución de la IF [dieciséis]. Además, los experimentos en ratones knockout NCX han demostrado actividad marcapasos ráfaga, lo que sugiere un posible papel contributivo de NCX en el sindrome taquicardia-bradicardia. Tanto HCN y NCX son responsables del "reloj de voltaje" de la actividad del marcapasos. Es posible que las disfunciones de las proteínas implicadas en el mecanismo de "reloj de calcio", por ejemplo, receptor de rianodina y retículo sarcoplásmico Ca2 p-ATPasa, también puede contribuir (Maltsev \& Lakatta, 2007)

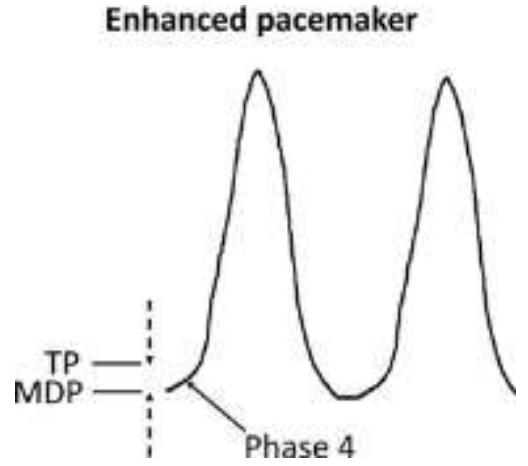

Figura 1. Marcapasos mejorado puede ocurrir a través de tres mecanismos: un cambio negativo en el umbral de potencial (TP), un cambio positivo en el potencial diastólico máximo (MDP), y un aumento de la frecuencia de la fase 4 de despolarización.

La automaticidad mejorada también puede ocurrir en el nodo AV, en condiciones de infarto agudo de miocardio, toxicidad digitalis, la administración iso-prenaline, y la cirugía cardíaca reciente. Cuando la velocidad de descarga del nodo AV es mayor que la frecuencia sinusal, puede conducir a los ritmos anormales llamadas ritmos de la unión acelerados. Estos ritmos pueden ocurrir en sitios cercanos a las aurículas, como en venas pulmonares, la vena cava 


\section{Mecanismos de las arritmias cardíacas}

Vol. 3, núm. 3., (2019)

Erick Hugo Zambrano Franco; Rossibell Berenisse Ollague Armijos; Betsy Elizabeth Quisilay Guamán; Lenin Israel Velasco Chávez

superior, cresta terminal, seno coronario, tabique auricular, y la región parahisiana que incluye la tricúspide y cánulas mitral, lo que lleva a la taquicardia auricular focal.

Alternativamente, la perisístole se produce cuando un marcapasos latente está protegida del marcapasos dominante por el bloque de entrada y se convierte, los potenciales de acción de descarga ectópicos independientemente. Una región de bloque de entrada se puede producir cuando el marcapasos dominante está rodeado por los tejidos isquémicos, infartado, o comprometidos de otro modo que impiden que la conducción de potenciales de acción al marcapasos latente. Cuando esto sucede, los potenciales de acción generados por el marcapasos latente pueden salir y activar el resto del miocardio. De este modo, se forma un foco para sistólico cuando hay tanto en la conducción comprometida en la conducción de marcapasos y salida ectópico.

La para sístole modulada es una variante de la anterior. Es el resultado de bloqueo de entrada incompleta del marcapasos ectópico. En esta situación, el marcapasos dominante o de otros tejidos cardíacos pueden ejercer influencia electro tónica en el foco para sistólico. Las influencias electro tónica que llegan temprano en el ciclo de marcapasos retrasan la fianillo del foco parasistolico, mientras que los que lleguen al final del ciclo aceleran su fianillo (Jalife, Antzelevitch, \& Moe, The case for modulated parasystole., 1982). Un caso especial de parasístole modulada se produce cuando los potenciales de acción del enfoque parasistolico ejercen influencia electro tónica en el enfoque en sí. Esto se denomina auto modulación.

\section{Actividad disparada}




\section{Mecanismos de las arritmias cardíacas}

Vol. 3, núm. 3., (2019)

Erick Hugo Zambrano Franco; Rossibell Berenisse Ollague Armijos; Betsy Elizabeth Quisilay Guamán; Lenin Israel Velasco Chávez

Resultados de la actividad desencadenada de la activación prematura de los tejidos cardíacos por posdespolarizaciones, que son las despolarizaciones provocadas por uno o más potenciales de acciónes anteriores.

\section{Posdespolarización precoz}

Posdespolarizaciones precoces (EAD por sus siglas en inglés) se pueden desarrollar antes de la plena repolarización, que corresponde a la fase 2 o fase 3 del potencial de acción cardíaca en seres humanos. Son por lo general, pero no exclusivamente asociadas con acción prolongada con potencial duracion (APDs), que se producen cuando la corriente de entrada es mayor en amplitud de la corriente hacia el exterior. Hay varios factores que pueden inclinar la balanza hacia la dirección hacia el interior. Estos incluyen el aumento de la corriente tardía de sodio (Ina), La corriente de calcio (Ical), o Incx, o disminuciones en las corrientes de repolarización de potasio (Ikr, Iks, Ik1). Se han propuesto dos mecanismos para la EAD que están asociados con prolongaciones en APDs y se producen durante la fase 2 del potencial de acción. En primer lugar, cambios despolarizantes en el potencial de membrana puede reactivar los canales de calcio de tipo L, lo que resulta en un aumento de Ica,l que además despolariza la membrana. Esto establece un bucle de retroalimentación positiva, lo que provoca un potencial de acción. En segundo lugar, en los potenciales de membrana negativos para el umbral de activación Ica,1 (pero antes de la plena repolarización), la liberación espontánea de calcio desde el retículo sarcoplásmico puede activar Incx, lo que resulta en la despolarización de la membrana. La naturaleza intermitente de las EAD ha sido recientemente examinada, demostrando que es debido a cambios lentos en [Nap]i y que podría explicar por qué las arritmias no ocurren todo el tiempo (Xie, Liao, \& Grandi, 2015). 


\section{Mecanismos de las arritmias cardíacas}

Vol. 3, núm. 3., (2019)

Erick Hugo Zambrano Franco; Rossibell Berenisse Ollague Armijos; Betsy Elizabeth Quisilay Guamán; Lenin Israel Velasco Chávez

EADS también se han asociado con la manteca en APDs, que se producen al final de la fase 3 del potencial de acción. Aquí, un APD abreviada permite la liberación normal de calcio desde el retículo sarcoplásmico. Si la concentración de calcio intracelular ([Ca2 p]i) Sigue siendo elevada cuando el potencial de membrana es negativo al potencial de equilibrio para NCX, Incx puede ser activada, provocando la despolarización de la membrana. Estos EAD finales son clínicamente relevantes, ya que pueden ocurrir inmediatamente después de la terminación de otros tipos de taquicardia, como la fibrilación absoluta, AT, TV y Fv. En tales casos, el tiempo de repolarización se acorta y una aumento transitorio en la liberación de calcio sarcoplásmico puede ser inducida al revertir a ritmo sinusal.

Cualquiera que sea el mecanismo subyacente, si el cambio en el potencial de membrana provocado por el EAD es suficientemente grande, se activará In/a, lo que resulta en la actividad desencadenada.

\section{Posdespolarizaciones retardadas}

Las posdespolarizaciones retardada (DADS) eran primero descritas como oscilamiento pospotenciales de reglamentación. Ellos se pueden desarrollar después de la plena repolarización, correspondiente a la fase 4 del potencial de acción cardíaca en seres humanos. DAD se observa en condiciones de sobrecarga de calcio intracelular, lo que puede resultar de la exposición a la digital, catecolaminas, hipokalemia, y la hipercalcemia, y en la hipertrofia e 


\section{Mecanismos de las arritmias cardíacas}

Vol. 3, núm. 3., (2019)

Erick Hugo Zambrano Franco; Rossibell Berenisse Ollague Armijos; Betsy Elizabeth Quisilay Guamán; Lenin Israel Velasco Chávez

insuficiencia cardíaca. El mecanismo propuesto para la génesis de las DAD es el siguiente: los altos niveles de calcio intracelular inducen la liberación espontánea de calcio desde el retículo sarcoplásmico, activando tres corrientes sensibles al calcio, la corriente catiónica no selectiva Ins, la corriente de intercambio sodio-calcio, Incx y la corriente de cloruro activada por calcio, Icl,cal. Juntos, estos constituyen el transitorio de corriente de entrada (ITI) que es responsable de la despolarización de la membrana (Guinamard, Chatelier, \& Demion, 2004). Si la despolarización producida por el DAD es sufficientemente grande, In/a se activa, lo que lleva a la actividad desencadenada. La actividad desencadenada inducida - DAD se cree que es la base de la arritmogénesis observado en la taquicardia ventricular poli-mórfica catecolaminérgicas (CPVT).

\section{La reentrada}

El reingreso se produce cuando un potencial de acción en sí no se apaga y se reactiva una región que se ha recuperado de refractariedad. Se puede dividir en dos tipos:

1. de reentrada que se produce en la presencia de un obstáculo, alrededor de la cual un potencial de acción puede viajar (tipo circo) la reentrada que se produce sin un obstáculo (reflexión o la fase 2).

2. Reentrada que implica un obstáculo (tipo de circo)

3. Esto ocurre cuando un potencial de acción viaja alrededor de un obstáculo anatómica o funcional y reexcites su lugar de origen.

\section{Reentrada que implica un obstáculo}




\section{Mecanismos de las arritmias cardíacas}

Vol. 3, núm. 3., (2019)

Erick Hugo Zambrano Franco; Rossibell Berenisse Ollague Armijos; Betsy Elizabeth Quisilay Guamán; Lenin Israel Velasco Chávez

La reentrada también puede ocurrir sin movimiento circular. Esto se puede dividir en reflexión y reentrada en fase 2 .

\section{Conclusiones.}

Los agentes antiarrítmicos novedosos que se han desarrollado son principalmente para el tratamiento de la fibrilación, la orientación de la aurícula específicanales iónicos c. Un ejemplo es vernakalant, un bloqueador de canales múltiples ultrarápida (IKur) Y acetilcolina-activado (IK, ACh) las corrientes de potasio. Esto tiene efectos mínimos en los ventrículos, y es por lo tanto menos probable de inducir arritmias ventriculares malignas. Sin embargo, no ha habido ningún fármaco recientemente autorizado para el tratamiento de las arritmias ventriculares, aunque ranolazina, un inhibidor retardado de In/a de la licencia para la angina, ha demostrado eficacia en estudios experimentales. Hay una necesidad de desarrollar nuevos medicamentos por varias razones. En primer lugar, cardioversión implantable desfibrilador (DAI) no son capaces de prevenir la aparición de arritmias ventriculares, pero sólo pueden poner fin a ellos, y no carecen de significación en la morbilidad. Sin embargo, ellos son la única forma de tratamiento demostrado que aumenta la esperanza de vida. Por otra parte, agentes farmacológicos actuales poseen efectos significativos secundarios cardiacos y extra cardíacas, el más preocupante de los cuales son sus propiedades proarrítmicas.

Una solución potencial es el diseño racional de agentes que se dirigen las proteínas anormales en cuestión. Por lo tanto, las EAD observadas en el síndrome de QT largo tipo 3 podrían prevenirse por las drogas que inhiben la tarde In/a. DAD, CPVT subyacentes pueden ser suprimidas por el bloqueo de los receptores de rianodina, que median la liberación de calcio 


\section{Mecanismos de las arritmias cardíacas}

Vol. 3, núm. 3., (2019)

Erick Hugo Zambrano Franco; Rossibell Berenisse Ollague Armijos; Betsy Elizabeth Quisilay Guamán; Lenin Israel Velasco Chávez

desde el retículo sarcoplásmico, o el intercambiador sodio-calcio. Por otra parte, la comprensión de la fisiología de arritmogenia, por ejemplo, los sustratos que sostienen la reentrada, pueden guiar el desarrollo de fármacos. Por lo tanto, las heterogeneidades en la acción potencial de conducción o la repolarización, observado en un número de canalopatías de iones, pueden ser reducidos por abridores de salida.

En resumen, este artículo revisa los mecanismos fisiológicos de arritmias por reentrada y no reentrada. Su generación y el mantenimiento son importantes en ambos síndromes arrítmicos congénitos y adquiridos. En el futuro, los fármacos antiarrítmicos tienen el potencial para que coincida con la terapia basada en dispositivo y la ablación con catéter en términos de eficacia.

\section{Bibliografía.}

Amos, G., Wettwer, E., \& Metzger, F. (1996). Differences between outward currents of human atrial and subepicardial ventricular myocytes. J Physiol, 15(491), 31-50.

Bolívar, J. (2015). Investigación Documental. México: Pax.

Carmeliet, E. (1999). Cardiac ionic currents and acute ischemia: from channels to arrhythmias. Physiol Rev, 917-1017.

Castro, J. (2016). Técnicas Documentales. México: Limusa.

Davila, A. (2015). Concepto de terminos cientificos. Caracas: Oasis.

Guinamard, R., Chatelier, A., \& Demion, M. (2004). Functional characterization of a Ca (2p)activated non-selective cation channel in human atrial cardiomyocytes. $J$ Physiol, 75-83.

Jalife, J., Antzelevitch, C., \& Moe, G. (1982). The case for modulated parasystole. Pacing Clin Electrophysiol, 911-26.

Jalife, J., Delmar, M., \& Anumonwo, J. (2009). Basic mechanisms of cardiac arrhyth-mias. Basic cardiac electrophysiology for the clinician. 2nd edWiley-Blackwell;:

Kanno, S., \& Saffitz, J. (2001). The role of myocardial gap junctions in electrical con-duction and arrhythmogenesis. Cardiovasc Pathol, 169-77. 


\section{Mecanismos de las arritmias cardíacas}

Vol. 3, núm. 3., (2019)

Erick Hugo Zambrano Franco; Rossibell Berenisse Ollague Armijos; Betsy Elizabeth Quisilay Guamán; Lenin Israel Velasco Chávez

Kunze, D., Lacerda, A., \& Wilson, D. (1985). Cardiac Na currents and the inacti-vating, reopening, and waiting properties of single cardiac Na channels. J Gen Physiol , 691719.

Maltsev, V., \& Lakatta, E. (2007). Normal heart rhythm is initiated and regulated by an intracellular calcium clock within pacemaker cells. Heart Lung Circ, 16(5), 335-48.

Weiss, J., Garfinkel, A., \& Karagueuzian, H. (2015). Perspective: a dynamics-based classification of ventricular arrhythmias. J Mol Cell Cardiol, 82(1), 136-52.

Xie, Y., Liao, Z., \& Grandi, E. (2015). Slow [Na]i changes and positive feedback between membrane potential and $[\mathrm{Ca}] \mathrm{i}$ underlie intermittent early after-depolarizations and arrhythmias. Circ Arrhythm Electrophysiol, 8(6), 1472-80. 